\title{
Psychosis patients refusing antipsychotic medicine could benefit from CBT in terms of both symptom reduction and social functioning
}

\author{
Tania Lecomte \\ Département de Psychologie, University of Montreal, Montreal, Quebec, Canada; tania.lecomte@umontreal.ca
}

\section{WHAT IS ALREADY KNOWN ON THIS TOPIC?}

Individuals with psychotic disorders might choose to forgo antipsychotic medication because of side effects. ${ }^{1}$ Over 40 randomised controlled trials (RCT) have demonstrated that cognitive behavioural therapy for psychosis (CBTp) is generally efficacious in improving symptoms, with small to medium effect sizes in individuals with psychotic disorders. ${ }^{2}$ It was unknown, until now, if individuals with psychotic disorders who were antipsychotic-free could tolerate CBTp and benefit from it.

\section{WHAT THIS PAPER ADDS?}

- This is the first RCT to demonstrate that individuals with schizophrenia-spectrum psychotic disorder receiving clinical services in first episode of psychosis programmes but refusing all antipsychotic medication could tolerate and benefit from CBTp both in terms of symptoms and social functioning.

- Morrison and colleagues offer insight into how symptoms change over 18 months in individuals not taking antipsychotics - whether receiving CBTp or otherwise - with few participants worsening overtime.

- Most participants in the CBTp condition improved in terms of overall and positive symptoms. It is noteworthy that these improvements were comparable to those found in other CBTp trials with individuals with psychosis taking medication and to those reported in antipsychotic medication trials.

\section{LIMITATIONS}

- The results can only be generalised to individuals with early psychosis and who are not in an acute psychotic state, receiving services but refusing medication, whereas many individuals with early psychosis refusing medication also refuse services.
- By only having a treatment as usual control condition, the study does not control for therapeutic effects of factors such as therapist attention or time in treatment.

- The treatment appeared to have stronger effects on cognitive versus emotional aspects of symptoms, suggesting that perhaps the treatment protocol should be revised to improve its impact on emotional aspects of psychosis.

\section{WHAT NEXT IN RESEARCH?}

Future RCTs should include a treatment-control condition and also assess dose-response (ie, how many sessions are needed to see improvements). Studies should also gather data on profiles of participants in order to determine who will be more or less likely to benefit from CBTp within this population.

\section{DO THESE RESULTS CHANGE YOUR PRACTICE AND WHY?}

My practice will not change since I already deliver CBTp to individuals who refuse their medication but who are open to receiving psychotherapy. These results will however help convince other health professionals to consider offering CBTp or referring to CBTp, their patients with psychosis who refuse to take their prescribed antipsychotic medication. This paper might also encourage more training of mental health workers in delivering CBTp to individuals presenting with psychotic symptoms.

Competing interests None.

doi:10.1136/eb-2014-101892

\section{REFERENCES}

1. Dibonaventura $\mathbf{M}$, Gabriel S, Dupclay L, et al. A patient perspective of the impact of medication side effects on adherence: results of a cross-sectional nationwide survey of patients with schizophrenia. BMC Psychiatry 2012;12:20.

2. Hofmann SG, Asnaani A, Vonk IJ, et al. The efficacy of cognitive behavioral therapy: a review of meta-analyses. Cognit Ther Res 2012;36:427-40.

ABSTRACT FROM: Morrison AP, Turkington D, Pyle M, et al. Cognitive therapy for people with schizophrenia spectrum disorders not taking antipsychotic drugs: a single-blind randomised controlled trial. Lancet 2014;383:1395-403.

Patients/participants Seventy-four people (age 16-65 years) who received an ICD-10 diagnosis of schizophrenia, schizoaffective disorder, delusional disorder or who met the entry criteria for an early intervention for psychosis service. Participants had to score at least four on the Positive and Negative Symptom Score (PANSS) for delusions or hallucinations or at least five for suspiciousness or persecution, conceptual disorganisation or grandiosity. All participants also had to have chosen not to take antipsychotic medication for the previous 6 months or decided never to take antipsychotics. The following exclusions applied: moderate to severe learning disability, organic impairment, lack of capacity to consent, acute inpatients, cognitive therapy in the previous 2 years and a primary diagnosis of substance or alcohol abuse.

Setting Manchester and Newcastle; February 2010 to May 2013.

Intervention Cognitive therapy plus treatment as usual $(\mathrm{n}=37)$. A total 26 weekly sessions were offered over 9 months and up to four booster sessions were available in the subsequent 9 months. Cognitive therapy was based on a specific cognitive module with an associated manual and delivered by therapists with specialist training who received regular supervision.

Comparison Treatment as usual $(\mathrm{n}=37)$.

Patient follow-up Assessments were three monthly; 81\% of participants (30 in each arm) were available for final assessment at 18 months.

Allocation Concealed.
Blinding Single blind (assessors).

\section{OUTCOMES}

Total PANSS At all follow-up times, cognitive therapy significantly reduced total symptoms compared to the treatment as usual group: estimated improvement -6.52 (95\% CI -10.79 to -2.25$)$. There was no overall deterioration in PANSS in either group (only two participants in each group had more than $50 \%$ deterioration in score over the course of the study).

PANSS positive At all follow-up times, cognitive therapy significantly improved positive symptom score compared to the treatment as usual group: estimated improvement -2.22 ( $95 \%$ CI -4.00 to -0.44 ).

PANSS negative Cognitive therapy did not have a significant effect on negative symptoms at any follow-up time: estimated improvement -1.02 ( $95 \%$ CI -2.35 to 0.30 ) compared with the treatment as usual group.

Adverse events There were eight serious events. In the cognitive therapy group, there was one overdose and one incident of a person deemed a risk to others. In the control group, there were two deaths (recorded as unrelated to the trial or mental health), three compulsory admissions and one attempted overdose. 